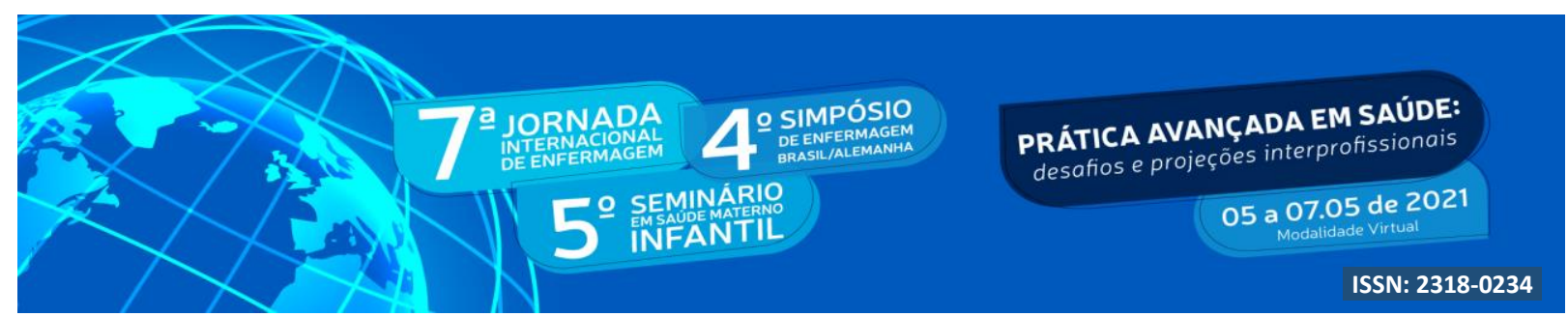

DOI: http://doi.org/10.48195/jie2021-021

\title{
ESPIRITUALIDADE NA PROMOÇÃO DO ENVELHECIMENTO E LONGEVIDADE: RELATO DE EXPERIÊNCIA ${ }^{1}$
}

\author{
Luane Sena²; Angélica Farinha³ ${ }^{3}$ Claudia Rivas; Renata Munhoz ${ }^{5}$; Naiana Oliveira dos \\ Santos ${ }^{6 ;}$ Maria Helena Gehlen ${ }^{7}$
}

\begin{abstract}
RESUMO
O estudo tem por objetivo descrever a espiritualidade na promoção do envelhecimento e longevidade com funcionalidade e bem-estar. Trata-se de um relato de experiência cujo processo foi vivenciado por acadêmicas de Enfermagem da Universidade Franciscana-UFN, no segundo semestre de 2019. O trabalho integrou as atividades curriculares da disciplina Atenção Integral à Saúde do Idoso. As informações foram coletadas através de uma entrevista semiestruturada, realizada com idosas moradoras da região central do estado do Rio Grande do Sul. O fenômeno do envelhecimento ocorre a nível mundial, garantir a essa população cuidados em saúde para um envelhecimento saudável necessita o acompanhamento da sociedade como um todo. Espiritualidade é um sentimento capaz de fazer suportar sentimentos, a relação entre espiritualidade e velhice se dá pela capacidade de suportar limitações inerentes ao processo. Conclui-se com esse estudo que o fenômeno do envelhecimento está relacionado a espiritualidade, trazendo uma nova percepção de vida.
\end{abstract}

Palavras-chave: Enfermagem; Longevidade; Idosos.

\begin{abstract}
The study aims to report the experience of academics when analyzing the understanding of the elderly in relation to spirituality. This is an experience report whose process was experienced by nursing students from the Franciscan University-UFN, in the second semester of 2019. The work integrated the curricular activities of the discipline Integral Attention to Health of the Elderly. The information was collected through a semi-structured interview, conducted with elderly women living in the central region of the state of Rio Grande do Sul. The phenomenon of aging occurs worldwide, guaranteeing this population health care for healthy aging requires the monitoring of society as a whole. Spirituality is a feeling capable of supporting feelings, the relationship between spirituality and old age is due to the ability to withstand limitations inherent in the process. It is concluded with this study that the phenomenon of aging is related to spirituality, bringing a new perception of life.
\end{abstract}

\footnotetext{
${ }^{1}$ Relato de Experiência

${ }^{2}$ Estudante do Curso de Enfermagem. Universidade Franciscana. E-mail: senaluane@ gmail.com

${ }^{3}$ Estudante do Curso de Enfermagem. Universidade Franciscana. E-mail: angelicaluciom2011@ hotmail.com

${ }^{4}$ Estudante do Curso de Enfermagem. Universidade Franciscana. E-mail: claudiamfrivas@gmail.com

${ }^{5}$ Estudante do Curso de Enfermagem. Universidade Franciscana. E-mail: renata09munhoz@gmail.com

${ }^{6}$ Professora Dra. do Curso de Enfermagem. Universidade Franciscana. E-mail: naiana.santos@ufn.edu.br

${ }^{7}$ Orientadora Dra. Professora do Curso de Enfermagem. Universidade Franciscana. E-mail: gehlenmh@gmail.com
} 


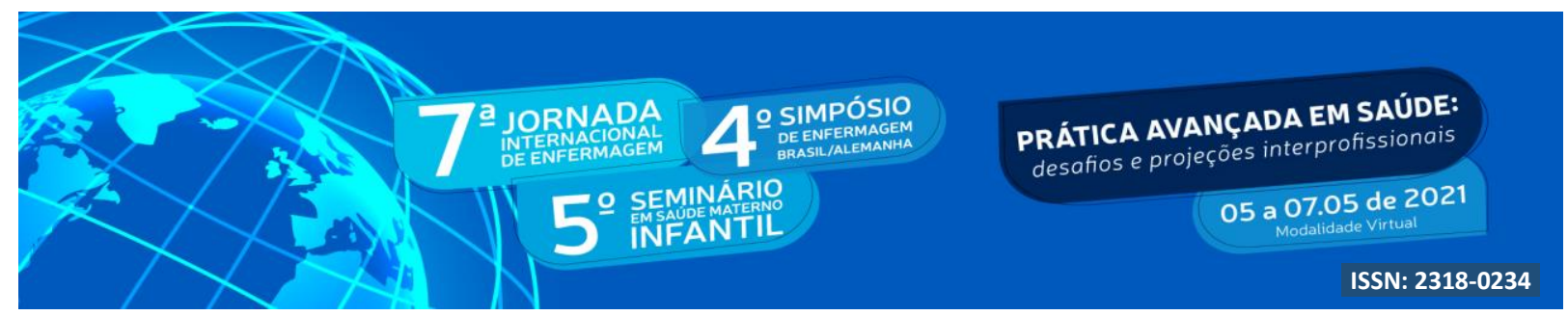

Key Words: Community; Family; Nursing.

\section{INTRODUÇÃ̃O}

O envelhecimento do ser humano é um processo natural, com gradativa redução do resguardo funcional, ao qual denominamos senescência. Em situações normais o envelhecimento não acarreta problemas ao indivíduo, porém situações de sobrecarga, de presença de patologias, acidentes e estresse pode levar a condições de adoecimento que necessitam de assistência, provocando o que chamamos senilidade (BRASIL, 2006).

O fenômeno do envelhecimento está ocorrendo a nível mundial e pode ser percebido no Brasil, resultado do aumento da expectativa de vida devido a melhores condições de saúde e pela diminuição da taxa de fecundidade feminina. Estima-se que em 2060, a população brasileira acima com 65 anos ou mais chegue a 25,5\% cerca de 58,2 milhões de idosos, em 2018 essa parcela populacional representava $9,2 \%$ ou seja 19,2 milhões de idosos (IBGE, 2018).

Com o advento de novas tecnologias e com o avanço da medicina espera-se o aumento significativo da longevidade humana (ALVES, 2015). O processo de envelhecimento com saúde encaminhará o indivíduo a uma longevidade sem maiores intercorrências (NASCIMENTO e SOUSA, 2015).

No cenário mundial atualmente encontra-se regiões onde as pessoas têm uma maior longevidade e uma melhor qualidade de vida, esses locais são denominados Blue Zones. Apesar de serem regiões com diferentes particularidades e com culturas diversificadas, há a semelhança de muitos idosos ultrapassarem os 100 anos de idade, com saúde, felicidade e longe de doenças tão presentes no nosso cenário atual. São lugares onde o estilo de vida é extraordinário e pessoas com mais de 105 anos aparentam ter 70. As populações apresentam pontos em comum, como atividade física, alimentação adequada e uma atitude positiva em relação à vida (LUGLIO, 2017).

Alves (2015), demonstra em seu estudo que envelhecimento e longevidade têm significados distintos, longevidade se refere às características ou qualidades do ser longevo, daquele que tem uma vida mais extensa que o esperado, já envelhecimento seria o ato de 


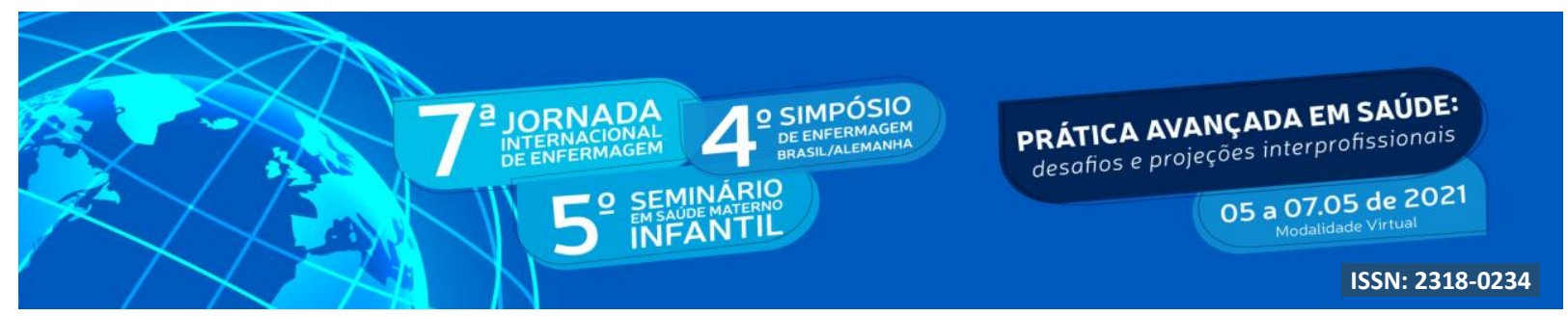

envelhecer, de se tornar velho.

Garantir a essa população cuidados em saúde para um envelhecimento saudável necessita o acompanhamento dos profissionais da saúde, dos gestores e da sociedade como um todo, tendo em vista que o problema primordial relacionado ao fenômeno do envelhecimento está no aumento de idosos senis (NASCIMENTO e SOUSA, 2015).

Espiritualidade é a disposição humana para encontrar um significado para a vida através de conceitos que ultrapassam o tangível, sentido de conexão com algo maior que si próprio, que pode ou não englobar uma participação religiosa formal (VOLCAN,2003).

Espiritualidade é aquilo que dá sentido à vida, e é um conceito mais amplo que religião pois esta é uma expressão da espiritualidade. Espiritualidade é um sentimento pessoal, que estimula um interesse pelos outros e por si, um sentido de significado da vida capaz de fazer suportar sentimentos debilitantes de culpa, raiva e ansiedade (SAAD; MASIERO; BATTISTELLA, 2001).

Diante do exposto, a espiritualidade na promoção do envelhecimento repercute na longevidade com funcionalidade e bem-estar auxiliando na percepção da multidimensionalidade dos cuidados destinados a essa parcela populacional.

\section{OBJETIVO}

Diante do exposto, objetiva-se descrever a espiritualidade na promoção do envelhecimento e longevidade com funcionalidade e bem-estar .

\section{METODOLOGIA}

Trata-se de um relato de experiência de uma atividade de ensino da disciplina atenção integral a saúde do idoso, cujo processo foi vivenciado por acadêmicas de Enfermagem da Universidade Franciscana, no decorrer do segundo semestre de 2019.

Assim os dados obtidos foram considerados mediante questionamentos elencados pelas acadêmicas, e pela própria observação e experiencia das mesmas. Sendo os 


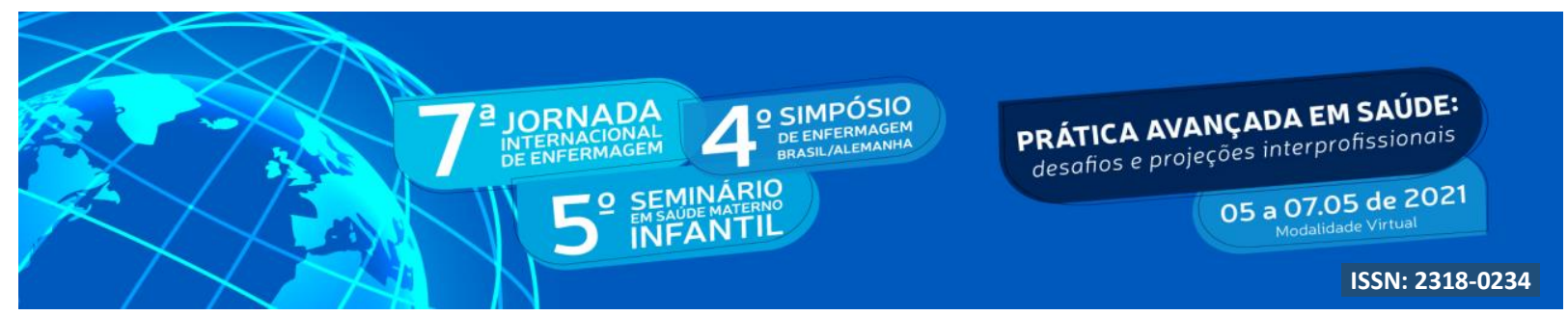

questionamentos: O que você considera por espiritualidade? Qual a importância da espiritualidade na longevidade?

Durante o processo de coleta de informações a singularidade das idosas foi valorizada em todos os seus aspectos, suas percepções e emoções, assim como suas compreensões sobre a sua condição de vida atual.

Após a coleta de dados, foi procedida as demais etapas do processo de enfermagem sendo que no planejamento houve uma análise interpretativa integrada com o aporte teórico o que permitiu determinar na implementação o estímulo da espiritualidade na promoção do envelhecimento com funcionalidade e bem-estar .

\section{RESULTADOS E DISCUSSÃO}

Embora atualmente o aumento da longevidade humana seja significativo, fazendo com que o idoso tenha uma expectativa de vida bem maior, o envelhecimento por ser um processo natural é inevitável e, em algum momento declínios fisiológicos vão atingir a todos que chegarem nessa etapa da vida, até mesmo os que sempre cuidaram da saúde e não possuem patologias.

Com análise no planejamento de enfermagem foi possível visualizar a importância que a espiritualidade tem no processo de envelhecimento com longevidade funcionalidade e bemestar, sendo uma implementação relevante para oferecer suporte emocional aos idosos que muitas vezes possuem fragilidades, angústias e medos nesta fase da vida, rodeada por incertezas como as idosas relataram nas entrevistas.

Neste sentido, a espiritualidade se torna uma dimensão a ser considerada na atenção integral a saúde da pessoa idosa em estímulo a longevidade. Evidenciou-se que a espiritualidade voltada para as práticas religiosas, devido a crença que a longevidade se relaciona com a da vida.

A longevidade é caracterizada por modificações morfológicas, funcionais, bioquímicas e psicológicas no ser humano, e é um determinante de perda progressiva da capacidade de 


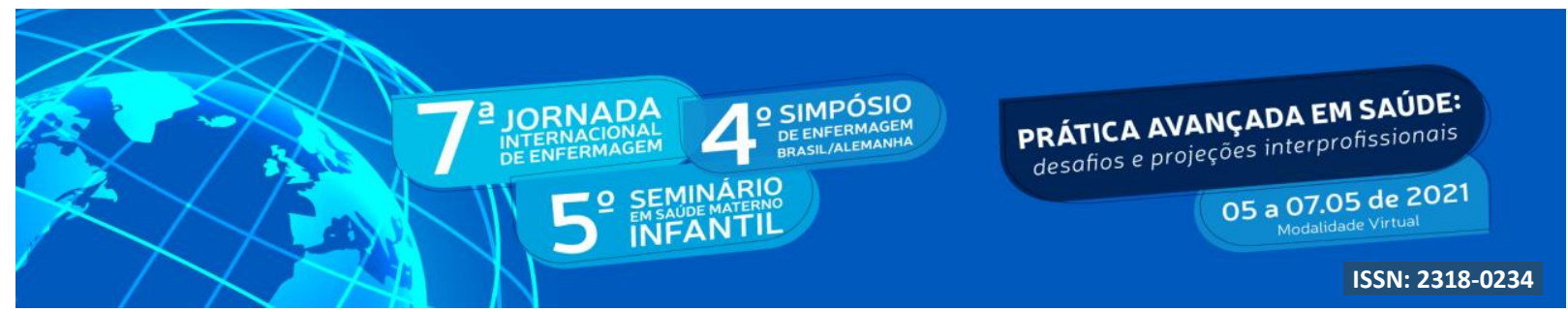

adaptação do indivíduo ao meio ambiente. Nessa fase da vida, os principais sistemas biológicos começam a apresentar declínios funcionais como a diminuição de força muscular, flexibilidade, agilidade, equilíbrio e capacidade cardiorrespiratória que com o tempo vão comprometer a execução das atividades de vida diárias (AVD's) necessárias para que o indivíduo viva de forma independente (OLIVEIRA, et.al.; 2015).

A diminuição do nível de atividade pode levar o idoso a um estado de fragilidade física e emocional e dependência para realizadas as atividades de vida diária, refletindo negativamente na qualidade de vida dos idosos, seja física, cognitiva ou psicológica. Nesses casos a espiritualidade se torna uma importante aliada, trazendo efeitos positivos no modo como os idosos vão enfrentar e aceitar essa fase da vida.

A atividade de ensino acerca da espiritualidade possibilitou o reconhecimento da dimensão espiritual no processo de envelhecimento como um estímulo a longevidade com conforto e bem-estar.

Nesse cenário, com o aumento da expectativa de vida, as pessoas idosas buscam estratégias para o enfrentamento do processo saúde-doença, visando melhoria da qualidade de vida e bem-estar na velhice. As estratégias de enfrentamento são definidas como "resiliência", que está relacionada à capacidade de recuperação e adaptação saudável diante de danos, adversidades e estressores (REIS; MENEZES; 2017).

Com o avançar da idade, a religiosidade e espiritualidade passam a representar uma importante ferramenta de suporte emocional, que reflete de forma significativa na saúde física e mental dessas pessoas (REIS; MENEZES; 2017).

Nem todos os idosos possuem o mesmo pensamento durante essa fase da vida, porém, em sua maioria, no decorrer desse processo de envelhecimento se consideram na última etapa da vida, e esse pensamento faz com que ocorra um aumento da frequência sobre o pensar na morte, a questão da finitude que parecia longínqua, pouco pensada, na velhice, torna-se mais próxima e até real (LUCCHETTI, et.al.; 2011).

Nesse processo, o retorno a uma prática religiosa passa a ser mais evidente e até indispensável, pois a espiritualidade é vista como uma busca pessoal que auxilia no 


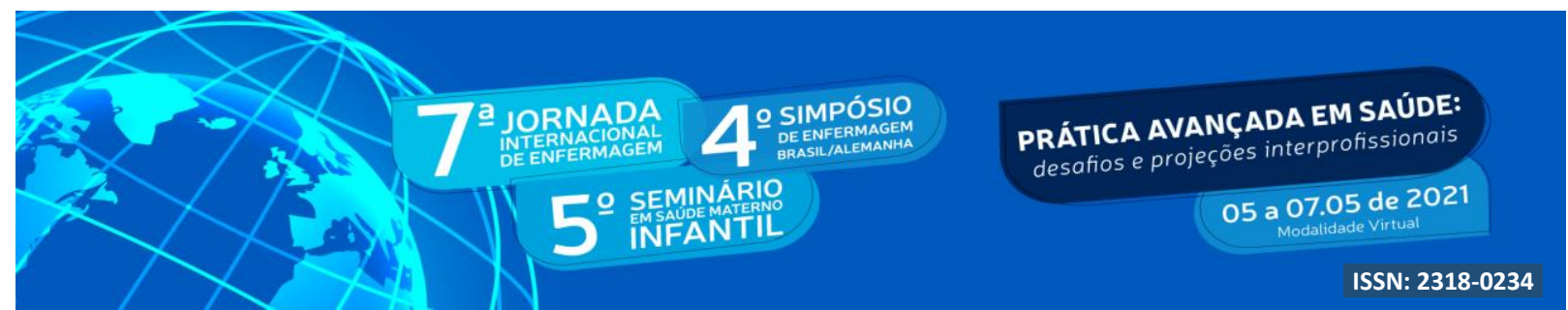

entendimento das questões finais sobre a vida, sobre seu sentido, sobre as relações com o sagrado ou transcendente (BRAVIN, et.al.; 2019).

Portanto, a relação entre espiritualidade e velhice se dá pela capacidade de suportar limitações, dificuldades e perdas inerentes ao processo, sendo que a vivência da espiritualidade possui importância e relevância para uma velhice com qualidade.

Dessa forma, a espiritualidade na promoção do envelhecimento com longevidade, funcionalidade e bem-estar mostrou-se como uma conquista, já que hoje em dia com a vida agitada e sempre movimentada, os indivíduos acabam esquecendo de realizar atividades que os proporciona prazer e bem-estar, aspectos esses de grande importância no processo de envelhecimento. Percebe-se, portanto, a importância da vivência pois a dimensão espiritual é também uma prática relevante para oferecer conforto aos idosos.

A percepção da espiritualidade como uma busca pela longevidade com funcionalidade e bem-estar oportunizou um melhor envelhecer, no sentido pessoal e existencial, explicitada na forma de gratidão a algo maior, transcendental, gratidão pela família, e possibilidade de continuar trabalhando, pela saúde, pelos ensinamentos realizados e por se sentir livre para realizar as suas vontades.

Portanto, a relação entre espiritualidade e velhice se dá pela capacidade de suportar limitações, dificuldades e perdas inerentes ao processo, sendo que a vivência da espiritualidade possui importância e relevância para a promoção do envelhecimento com uma velhice com funcionalidade.

\section{CONCLUSÃO}

Ao descrever a espiritualidade na promoção do envelhecimento e longevidade com funcionalidade e bem-estar, reforçou os pressupostos acadêmicos de que o envelhecimento é um processo natural e complexo e que a percepção do envelhecer está relacionada a espiritualidade, evidenciando que envelhecer traz consigo uma nova perspectiva na maneira de sentir a vida, seja com mais gratidão, com a sensação de dever cumprido e com uma ligação mais íntima com algo superior. 


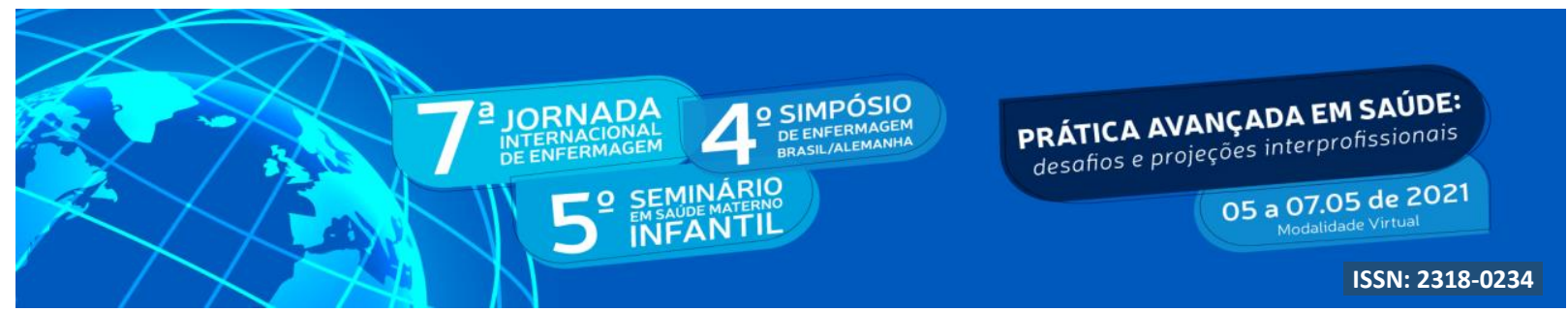

Perceber os idosos de forma multidimensional, é transpor a visão reducionista que enfoca apenas o aspecto clínico e por vezes patológico. É necessário conhecer as transformações fisiológicas decorrentes desse processo, ter uma conduta apropriada e principalmente observar todas as dimensões da vida, desde aspectos psicossociais, educacionais, funcionais até a dimensão espiritual e sua importância para um envelhecimento saudável.

Sendo assim, o desenvolvimento deste estudo trouxe uma ampliação do saber, no sentido que permitiu entender que a espiritualidade é imprescindível para o envelhecer e consequentemente para atingir a longevidade com funcionalidade.

\section{REFERÊNCIAS}

ALVES, J. E. D. Envelhecimento, Longevidade e Longevidade da População Brasileira. Longevidade ADunicamp, 2015.

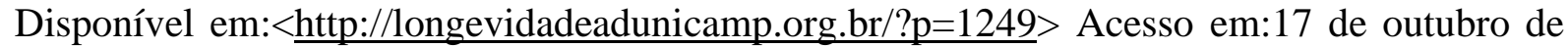
2019.

BRASIL, Ministério da Saúde. Envelhecimento e Saúde da Pessoa Idosa. Secretaria de Atenção à Saúde Departamento de Atenção Básica, Cadernos de Atenção Básica - n. ${ }^{\circ}$ 19, Brasília/DF; 2006.

BRAVIN, A.M.; TRETTENE, A.S.; ANDRADE, L.G.M.; POPIM, R.G. Benefícios da espiritualidade e/ou religiosidade em pacientes renais crônicos: revisão integrativa. Rev Bras Enferm. v. 72, n. 2, p. 568, 2019.

IBGE. Instituto Brasileiro de Geografia e Estatística. Número de idosos cresce $18 \%$ em 5 anos e ultrapassa 30 milhões em 2017. Estatísticas Sociais, 2018.

Disponível em: <https://agenciadenoticias.ibge.gov.br/agencia-noticias/2012-agencia-denoticias/noticias/20980-numero-de-idosos-cresce-18-em-5-anos-e-ultrapassa-30-milhoes-em2017> Acesso em 17 de outubro de 2019.

LUGLIO, A. Blue Zones: Os lugares onde as pessoas vivem mais. Estadão, Portal do Estado de S. Paulo, 2017.

LUCCHETTI, G.; LUCCHETTI, A.L.G.; BASSI, R.M.; NASRI, F.; NACIF, S.A.P.; O idoso e sua espiritualidade: impacto sobre diferentes aspectos do envelhecimento. Rev. Bras. Geriatria e Gerontologia, vol. 14, n. 1, p. 2, 2011. 


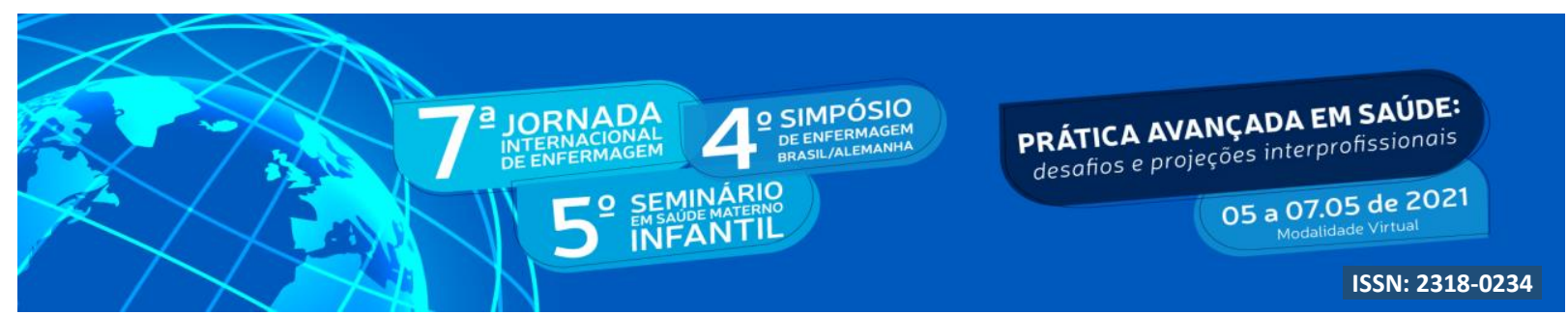

NASCIMENTO, D.S. SOUSA, I.P.C. Longevidade Saudável: O Desafio da Enfermagem, $4^{\circ}$ CIEH Congresso Internacional de Envelhecimento Humano. Anais CIEH, v. 2, n.1, p.17,2015 .

NEVES, J.L. Pesquisa Qualitativa-Características, usos e possibilidades. Caderno de pesquisas em administração. v. 1, n. 3, p. 1-5, 1996.

OLIVEIRA, J.M.; FERREIRA, S.O.; BISPO, N.N.C.; CONCONE, M.H.V.B.; Alterações físicas decorrentes do envelhecimento na perspectiva de idosos institucionalizados. Revista Kairós Gerontologia, vol. 18, n. 4, p. 4, 2015.

REIS, L.A.; MENEZES, T.M.O.; Religiosidade e espiritualidade nas estratégias de resiliência do idoso longevo no cotidiano. Rev Bras Enferm, vol. 70, n. 4, p. 2, 2017.

SAAD, M.; MASIERO, D.; BATTISTELLA, L. Espiritualidade baseada em evidências. Acta Fisiátrica, v. 8, n. 3, p. 107-112, 2001.

VOLCAN, S.M.A. - Relationship between spiritual well-being and minor psychiatric disorders: cross-sectional study. Rev. Saúde Pública, v.37, n. 4, p. 440-445, 2003. 